\title{
Heterogeneous Flow During High-Pressure Torsion
}

\author{
Roberto B. Figueiredo ${ }^{\mathrm{a} *}$, Terence G. Langdon ${ }^{\mathrm{b}, \mathrm{c}}$ \\ ${ }^{a}$ Department of Materials Engineering and Civil Construction, \\ Universidade Federal de Minas Gerais - UFMG, CEP 31270-901, Belo Horizonte, MG, Brazil \\ ${ }^{\mathrm{b}}$ Departments of Aerospace \& Mechanical Engineering and Materials Science, \\ University of Southern California, 90089-1453, Los Angeles, CA, U.S.A. \\ ${ }^{\mathrm{c}}$ Materials Research Group, Faculty of Engineering and the Environment, \\ University of Southampton, Southamton SO17 1BJ, U.K
}

Received: September 11, 2012; Revised: October 24, 2012

\begin{abstract}
High-Pressure Torsion (HPT) has attracted significant attention in recent years as an effective technique to process ultrafine and nanostructured materials. The hydrostatic pressure developed during processing prevents the occurrence of cracks and the low thickness to diameter ratio provides the opportunity for developing high strains at low numbers of rotations. The present work analyses the plastic flow during HPT. Experimental results and computer modeling are used to describe heterogeneous plastic flow. It is shown that variations in structure, hardness and in the distribution of strain are observed along the disc thickness. The sources of these heterogeneities are discussed.
\end{abstract}

Keywords: high-pressure torsion, plastic flow, heterogeneous flow

\section{Introduction}

Severe plastic deformation (SPD) is now a widely recognized tool to refine the grain structure of metallic materials to the submicrometer, or even nanometer, range. High-pressure torsion (HPT) ${ }^{1}$ is one of the most used SPD processing techniques. During HPT a sample in the shape of a thin disc is placed between massive rigid anvils and subjected to a compression stress. One of the anvils rotates in relation to the other and the contact friction between the anvils and the sample leads to torsional deformation of the disc.

The structure and the mechanical properties are found to depend strongly on the level of imposed strain during HPT. Usually the grain size decreases and the strength increases with increasing strain. The imposed strain, $\varepsilon$, is usually calculated as in the Equation $1^{2,3}$ :

$\varepsilon=\frac{2 \pi N r}{t \sqrt{3}}$

where $N$ is the number of turns, $r$ is the distance to the center of the disc and $t$ is the thickness of the disc. Thus, it follows that the level of strain, and consequently the grain refinement and the increase in strength, are expected to be higher at the edge of the disc and after larger numbers of turns. Moreover, it is expected that the strain does not vary either along the disc through-thickness direction or with varying imposed compression stress. Many papers have confirmed the expected trend of increasing hardness with increasing distance to the center of the sample at low numbers of rotations ${ }^{4-9}$. However the theoretical prediction is not always confirmed. There are reports of variations in the distributions of microhardness with variations in the applied

*e-mail: figueiredo-rb@ufmg.br pressure $e^{3,5,6}$ and variations of hardness in the throughthickness direction ${ }^{10,11}$. Thus, the theoretical description of plastic flow is not always accurate and a more detailed analysis is needed.

The present paper aims to show that heterogeneous flow takes place during HPT in certain conditions. The plastic flow is indirectly evaluated by the evolution of grain structure and microhardness and directly evaluated using finite element modeling (FEM).

\section{Experimental Procedure}

The material used in the experiments was a $\mathrm{Mg}-3 \%$ Al-1\% Zn alloy (AZ31) provided by Timminco Co. (Aurora, $\mathrm{CO}$ ) as extruded rods with $\sim 10 \mathrm{~mm}$ diameter. Discs with $\sim 1.5 \mathrm{~mm}$ thickness were cut from the rods and ground to $\sim 0.8 \mathrm{~mm}$ thickness using abrasive grids. The discs, with $10 \mathrm{~mm}$ diameter and $0.8 \mathrm{~mm}$ thickness, were processed by HPT under quasi-constrained condition ${ }^{1}$ at both room temperature and at $463 \mathrm{~K}$. Small heating elements were placed around the anvils of the equipment in order to reach the high temperature. Processing was carried out under $6 \mathrm{GPa}$ of nominal pressure. The rotation rate was $1 \mathrm{rpm}$. Multiple discs were processed to total numbers of turns, $N$, between $1 / 4$ and 5 .

Following processing, the discs were cut and mounted for grinding and polishing of the longitudinal sections. Conventional metallographic techniques were used and the final polishing of the surfaces was carried out using a colloidal silica solution. Microhardness tests were carried out along the longitudinal section using a Vickers indenter. Details of the HPT processing, sample preparation and microhardness tests are given elsewhere ${ }^{12,13}$. 


\section{Modeling}

Finite element modeling, in both $2 \mathrm{D}$ and $3 \mathrm{D}$, was carried out using DEFORM software version 10.0. The model considered the geometry of quasi-constrained HPT in which a thin disc, with $0.8 \mathrm{~mm}$ thickness and $10 \mathrm{~mm}$ diameter, is placed between rigid anvils containing a shallow depression with depth of less than half the thickness of the disc. Therefore, the anvils do not touch each other during processing and there is some material flow in the small gap between the anvils. The sample was modeled as a rigid-plastic object and the anvils were considered rigid objects. The mesh had 2,000-3,000 elements in the $2 \mathrm{D}$ and $\sim 35,000$ elements in the 3D simulations. A sticking condition was considered in the contact between the sample top and bottom surfaces and the anvil depression surfaces. A compression force was applied to the sample by the top anvil and the bottom anvil rotated with a constant rotation rate of $1 \mathrm{rpm}$. The simulations considered materials with strainhardening, perfect-plastic and flow-softening behavior. The former is expected to correlate to the behavior of metallic materials deformed at low temperatures while perfect-plastic and flow-softening behavior correlate to deformation at high temperatures. Details of the simulation are given elsewhere ${ }^{14,15}$

\section{Results}

\subsection{Structural evolution}

The longitudinal section of the HPT processed discs exhibits inhomogeneous features along the throughthickness direction. Figure 1 shows a representative image of the edge of a disc processed by 5 turns at room temperature.

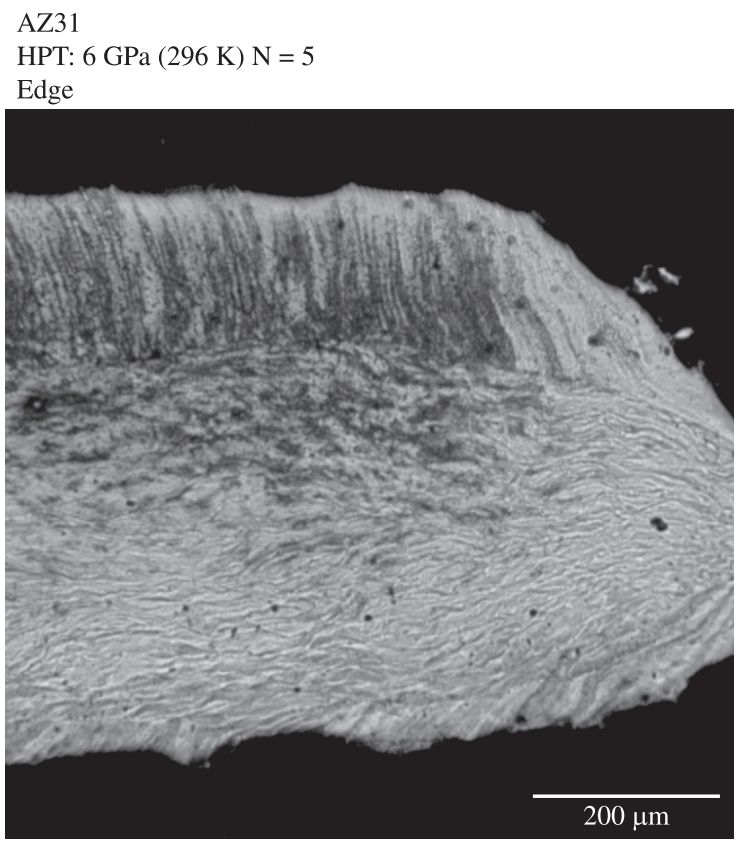

Figure 1. Low magnification image of the edge of the longitudinal section of a disc processed by 5 turns of HPT at room temperature ${ }^{13}$.
The sample top and bottom surfaces are parallel to the horizontal direction of the image. It is clearly observed that the top region of the disc exhibits a more distinct structural pattern than the middle and bottom region. Nearly vertical lines are observed in the top area while discontinuous and nearly horizontal lines are observed in the other areas.

The vertical lines observed in Figure 1 are also present in the as-received material due to the previous extrusion process. However, HPT deformation is expected to change this pattern to horizontal lines since the shear plane is horizontal. Thus, the heterogeneous features observed along the thickness of the sample are attributed to a difference in the deformation level. The top region of the disc maintains the orientation of the asreceived material suggesting a low level of deformation while the bottom area attains a different orientation of structural features suggesting a high deformation level. The image in Figure 1 also shows asymmetry on the overall shape of the disc. This is attributed to minor misalignment of the anvils during processing at the high pressure of $6 \mathrm{GPa}$.

The grain structure of a sample processed by HPT for $1 / 4$ turn at $463 \mathrm{~K}$ is shown in Figure 2. The bottom surface of the sample is parallel to the horizontal direction of the

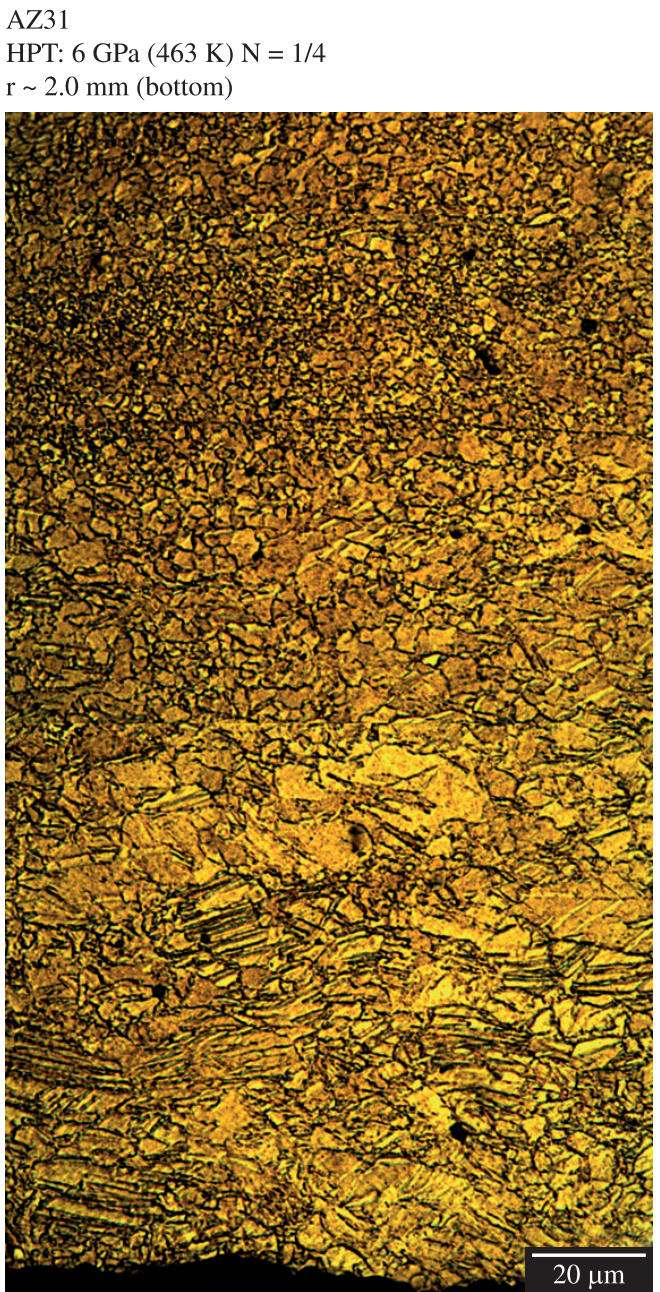

Figure 2. Grain structure in the region near the bottom of a sample processed by HPT for $1 / 4$ turn at $463 \mathrm{~K}^{12}$. 
image. It is observed that the grain size varies significantly along the top-bottom direction. Coarse grains with twins are observed at the bottom of the sample while small refined grains are observed in the top of the image. Early papers have shown that grain refinement in magnesium alloys in this temperature range depends on the level of imposed strain $^{16-18}$. In practice a very large level of strain is required for homogeneous grain refinement while low strains lead to partial grain refinement and a heterogeneous grain size distribution. Thus, the grain structure exhibited in Figure 2 suggests that a high strain was imposed on the top region while a low strain was imposed to the bottom region.

\subsection{Microhardness distribution}

Figure 3 shows a color-coded distribution of hardness along the half longitudinal sections of discs of magnesium alloy AZ31 after being processed by HPT at $463 \mathrm{~K}$ for different numbers of turns. It is observed that the hardness variation is more significant in the top-bottom direction than at different distances from the center. Higher levels of hardness are observed around the mid-plane in the samples processed by $1 / 4$ and 1 turn of HPT and near the bottom area in the sample processed by 5 turns. It is worth noting that the distribution of hardness in the sample processed by 1/4 turn agrees with the grain structure shown in Figure 2. Lower hardness is observed near the bottom of the disk at a distance of $\sim 2 \mathrm{~mm}$ from the center compared to the region at $\sim 200 \mu \mathrm{m}$ from the bottom. The higher hardness at a moderate distance from the bottom agrees with the smaller grain sizes in this region compared to the region nearer to the bottom.

\subsection{Finite element modeling}

The appearance of the sample at different stages of deformation during one turn of HPT is shown in Figure 4. The simulation considered a constant rotation rate of $1 \mathrm{rpm}$. The top anvil was removed for the images to allow visualization of the sample. It is observed that at the very early stage of deformation the sample attains the shape of the anvil depression and there is a small thickness reduction. At $1 / 4$ turn (15 seconds) there is a noticeable material outflow around the edge of the sample in the form of a thin ribbon between the anvils. This material outflow increases with increasing rotation and leads to reduction of the sample thickness.

Figure 5 shows the general distribution of effective strain on the longitudinal section of samples with different initial thicknesses after processing by HPT to different numbers of turns. It is observed there is a clear trend of increasing strain with increasing distance from the center of the samples. The distribution of strain is fairly homogeneous along the through-thickness direction except for the region near the edge of the discs and in the sample with the largest initial thickness.

Figure 6 shows the distribution of strain-rate during HPT processing considering different levels of friction on the top and bottom anvil walls. The top three images show the distributions during simulations in which both anvils exhibit similar friction levels and the bottom two show images of simulations in which the friction level is dissimilar in both anvils. It is observed that the plastic flow is not homogeneous along the through-thickness direction

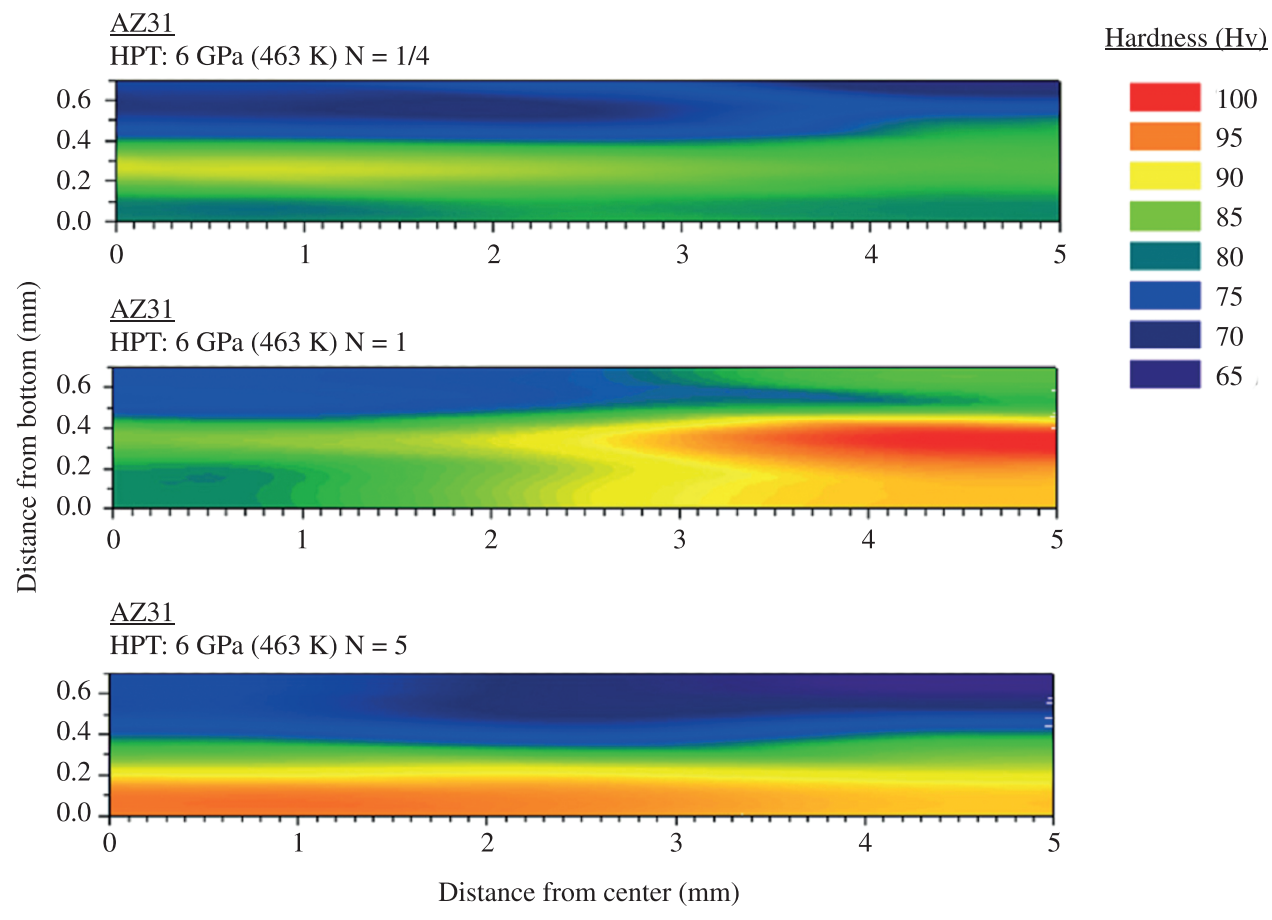

Figure 3. Color-coded distribution of hardness along the half longitudinal sections of discs of magnesium alloy AZ31 after being processed by HPT at $463 \mathrm{~K}$ for (top) $1 / 4$ turn, (middle) 1 turn and (bottom) 5 turns $^{12}$. 


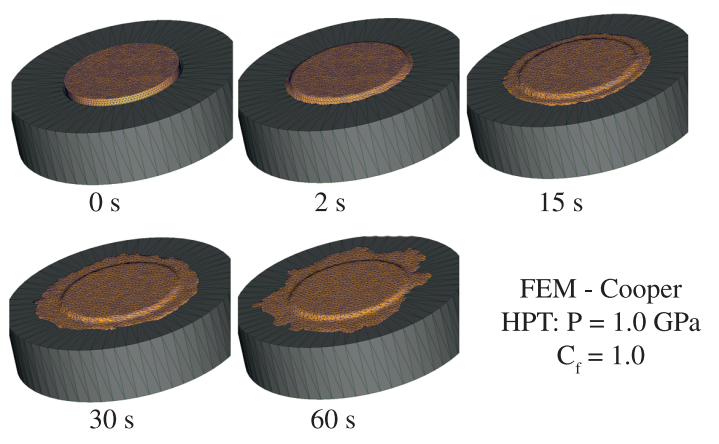

Figure 4. Appearance of the sample at different stages of deformation during one turn of $\mathrm{HPT}^{14}$.

FEM - HPT

Strain-hardening material

Initial diameter: $10 \mathrm{~mm}$

Initial

thickness (mm):

(a) 0.8 $\mathrm{N}=1$

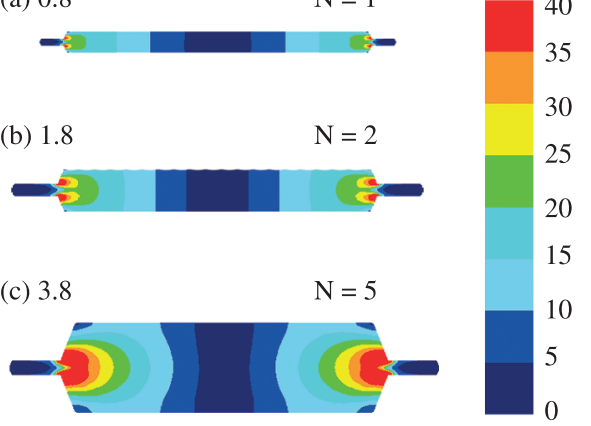

Figure 5. Distribution of effective strain on the longitudinal section of samples with initial thickness of (a) $0.8 \mathrm{~mm}$, (b) $1.8 \mathrm{~mm}$ and (c) $3.8 \mathrm{~mm}$ after processing by $\mathrm{HPT}^{15}$.

FEM - HPT

Perfect-plastic material

Friction coefficient:

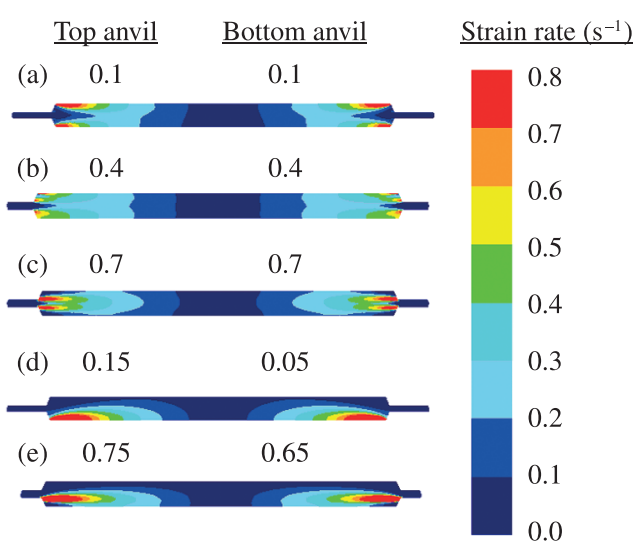

Figure 6. Distribution of strain-rate during HPT processing considering different levels of friction on the top and bottom anvils' walls ${ }^{15}$. and the variations in the rate of flow are more significant at the edge of the samples. Moreover, the concentration of plastic flow depends strongly on the friction with the lateral walls of the anvils. For conditions of low friction, the plastic flow concentrates near the top and bottom surfaces of the samples while it concentrates near the mid-plane when friction is high. This leads to the formation of an area with low deformation on the top and bottom corners of the sample. It is also shown that when friction is dissimilar on both anvils the plastic flow concentrates near the anvil with lower friction. Figure 6d shows flow localization near the bottom surface while Figure 6e shows flow localization between the mid-plane and the bottom surfaces. This leads to the formation of an area of low strain near the bottom surface corner. The formation of this area of low deformation is similar to that observed in Figure $6 c$ except that the latter also exhibits deformation near the top anvil.

Figure 7 shows the distribution of mean stresses during simulations that considered different levels of nominal compression stresses. The nominal compression stresses were calculated as the compressive force applied by the anvils divided by the initial area of the sample. It is observed that the level of mean stresses varies linearly with the distance from the center of the disc. Larger compressive stresses are observed near the center compared to the edge of the discs. In practice, compressive stresses in the range of the nominal stresses are only observed in a small region around the center of the disc while lower values are observed near the edge.

\section{Discussion}

The results reported in the present paper confirm the occurrence of flow heterogeneities during HPT. The flow lines at the edge in the longitudinal section of a disc of magnesium alloy processed by HPT (Figure 1) reveal the existence of areas with distinct plastic flow along the through-thickness direction. This is confirmed by the grain structure depicted in Figure 2 for an area away from the edge of the disc and by the microhardness distribution along the longitudinal section of discs processed to different numbers of turns (Figure 3). It is worth noting that areas of low deformation may be observed near the top anvil (Figures 1 and 3) or near the bottom anvil (Figure 2). This shows that flow concentration does not follow a clear trend in relation to the static (top) or the rotating (bottom) anvil. It depends solely on random variations in friction coefficients between the top and bottom anvils.

These heterogeneities revealed by experiments are not predicted by the theory of plastic flow during HPT. Similar findings were reported for aluminum ${ }^{10}$ and for iron ${ }^{11}$ samples with low aspect ration. However, microhardness distributions at different distances from the bottom of samples of high aspect ratio of pure aluminum revealed homogeneous flow ${ }^{19}$. Thus, the present results suggest that heterogeneous plastic flow during HPT depends on both sample aspect ratio and material behavior.

Since the theoretical description failed to explain the heterogeneous plastic flow and the influence of other processing parameters like nominal pressure and friction between the sample and the anvils, FEM was carried out. The 


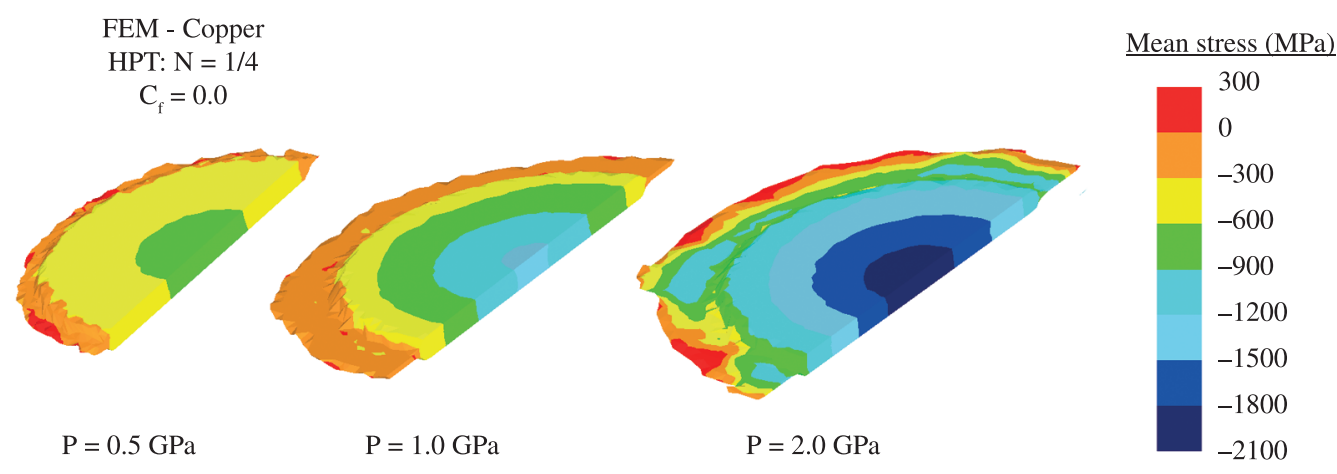

Figure 7. Distribution of mean stresses during simulations that considered different levels of nominal compression stresses ${ }^{14}$.

results show the material outflow during HPT considering quasi-constrained conditions and also show variations in the mean stresses along the sample which had not been predicted earlier.

Also, FEM successfully predicts heterogeneous plastic flow along the through-thickness direction of discs during HPT. The more pronounced heterogeneities are observed at the edge of the samples and in samples with high thicknessto-diameter ratio. FEM simulations revealed that friction between the samples and the lateral walls of the anvils play a significant role in plastic flow during HPT. Friction with the walls was considered as the source of heterogeneous deformation at the edge of samples of iron ${ }^{11}$ and a recent report ${ }^{20}$ describes the formation of a dead metal zone in this area due to friction in a sample of a magnesium alloy.

\section{Summary and Conclusions}

- The results of structural characterization, microhardness tests and finite element modeling

\section{References}

1. Zhilyaev AP and Langdon TG. Using high-pressure torsion for metal processing: Fundamentals and applications. Progress in Materials Science. 2008; 53:893-979. http://dx.doi. org/10.1016/j.pmatsci.2008.03.002

2. Valiev RZ, Ivanisenko YV, Rauch EF and Baudelet B. Structure and deformation behavior of Armco iron subjected to severe plastic deformation. Acta Materialia. 1996; 44:4705-4712. http://dx.doi.org/10.1016/S1359-6454(96)00156-5

3. Zhilyaev AP, Nurislamova GV, Kim B-K, Baró MD, Szpunar JA and Langdon TG. Experimental parameters influencing grain refinement and microstructural evolution during highpressure torsion. Acta Materialia. 2003; 51:753-765. http:// dx.doi.org/10.1016/S1359-6454(02)00466-4

4. Vorhauer A and Pippan R. On the homogeneity of deformation by high pressure torsion. Scripta Materialia. 2004; 51:921-925. http://dx.doi.org/10.1016/j.scriptamat.2004.04.025

5. Xu C, Horita Z and Langdon TG. The evolution of homogeneity in an aluminum alloy processed by high-pressure torsion. Acta Materialia. 2008; 56:5168-5176. http://dx.doi.org/10.1016/j. actamat.2008.06.036

6. Xu C and Langdon TG. Three dimensional representations of hardness distributions after processing by high-pressure torsion. Materials Science and Engineering. 2009; A503:71-74.
(FEM) reveal heterogeneous flow during processing by HPT;

- Variations of grain structure, microhardness and distribution of strain are observed along the throughthickness direction of discs;

- The sample aspect ratio and friction between the sample and the lateral walls of the anvils during processing are the main sources of heterogeneous flow; and

- Material outflow between the anvils and variations in the distributions of mean stresses during HPT are predicted by FEM.

\section{Acknowledgements}

This work was supported in part by the Brazilian Research Council (CNPq) under Grant Agreement No. 483077/2011-9 and in part by the European Research Council under ERC Grant Agreement No. 267464-SPDMETALS.

7. Loucif A, Figueiredo RB, Baudin T, Brisset F and Langdon TG. Microstructural evolution in an Al-6061 alloy processed by high-pressure torsion. Materials Science and Engineering. 2010; A527:4864-4869. http://dx.doi. org/10.1016/j.msea.2010.04.027

8. Wongsa-Ngam J, Kawasaki M, Zhao Y and Langdon TG. Microstructural evolution and mechanical properties of a $\mathrm{Cu}$ $\mathrm{Zr}$ alloy processed by high-pressure torsion. Materials Science and Engineering. 2011; A528:7715-7722.

9. Kawasaki M, Alhajeri SN, Xu C and Langdon TG. The development of hardness homogeneity in pure aluminum alloy disks processed by high-pressure torsion. Materials Science and Engineering. 2011; A529:345-351.

10. Sakai G, Nakamura K, Horita Z and Langdon TG. Developing high-pressure torsion for use with bulk samples. Materials Science and Engineering. 2005; A406:268.

11. Hohenwarter A, Bachmaier A, Gludovatz B, Scheriau $\mathrm{S}$ and Pippan R. Technical parameters affecting grain refinement by high pressure torsion. International Journal of Materials Research. 2009; 100:1653-1661. http://dx.doi. org/10.3139/146.110224

12. Figueiredo RB and Langdon TG. Development of structural heterogeneities in a magnesium alloy processed by high- 
pressure torsion. Materials Science and Engineering. 2011; A528:4500-4506.

13. Figueiredo RB, Aguilar MTP, Cetlin PR and Langdon TG. Deformation heterogeneity on the cross-sectional planes of magnesiumalloy processed by high-pressure torsion. Metallurgical and Materials Transactions. 2011; 42A:3013-3021. http:// dx.doi.org/10.1007/s11661-011-0609-Z

14. Figueiredo RB, Cetlin PR and Langdon TG. Using finite element modeling to examine the flow process in quasiconstrained high-pressure torsion. Materials Science and Engineering. 2011; A528:8198-8204.

15. Figueiredo RB, Aguilar MTP, Cetlin PR and Langdon TG. Analysis of plastic flow during high-pressure torsion. Journal of Materials Science. 2012; 47:7807-7814. http://dx.doi. org/10.1007/s10853-012-6506-z

16. Figueiredo RB and Langdon TG. Principles of grain refinement in magnesium alloys processed by equal-channel angular pressing. Journal of Materials Science. 2009; 44:4758-4762. http://dx.doi.org/10.1007/s10853-009-3725-z

17. Figueiredo RB and Langdon TG. The nature of grain refinement in equal-channel angular pressing: a comparison of representative fcc and hcp metals. International Journal of Materials Research. 2009; 100:1638-1646. http://dx.doi. org/10.3139/146.110228

18. Figueiredo RB and Langdon TG. Grain refinement and mechanical behavior of a magnesium alloy processed by ECAP. Journal of Materials Science. 2010; 45:4827-4836. http:// dx.doi.org/10.1007/s10853-010-4589-y

19. Kawasaki M, Figueiredo RB and Langdon TG. An investigation of hardness homogeneity throughout disks processed by highpressure torsion. Acta Materialia. 2011; 59:308-316. http:// dx.doi.org/10.1016/j.actamat.2010.09.034

20. Lee DJ, Yoon EY, Park LJ and Kim HS. The dead metal zone in high-pressure torsion. Scripta Materialia. 2012; 67:384-387. http://dx.doi.org/10.1016/j.scriptamat.2012.05.024 